\title{
A maximin characterisation of the escape rate of non-expansive mappings in metrically convex spaces
}

\author{
Stéphane Gaubert* Guillaume Vigeral ${ }^{\dagger}$
}

August 1, 2011

\begin{abstract}
We establish a maximin characterisation of the linear escape rate of the orbits of a non-expansive mapping on a complete (hemi-)metric space, under a mild form of Busemann's non-positive curvature condition (we require a distinguished family of geodesics with a common origin to satisfy a convexity inequality). This characterisation, which involves horofunctions, generalises the Collatz-Wielandt characterisation of the spectral radius of a non-negative matrix. It yields as corollaries a theorem of Kohlberg and Neyman (1981), concerning non-expansive maps in Banach spaces, a variant of a Denjoy-Wolff type theorem of Karlsson (2001), together with a refinement of a theorem of Gunawardena and Walsh (2003), concerning order-preserving positively homogeneous self-maps of symmetric cones. An application to zero-sum stochastic games is also given.
\end{abstract}

\section{Introduction}

A self-map $T$ of a metric space $(X, d)$ is non-expansive if $d(T(x), T(y)) \leqslant d(x, y)$. A general problem consists in studying the asymptotic behaviour of the orbits of $T$. This is motivated in particular by the celebrated theorem of Denjoy and Wolff [Den26. Wol26a, Wol26b on the iteration of holomorphic self-maps of the unit disk (these maps are non-expansive in Poincare's metric). Other motivations arise from the cases of non-expansive self-maps of Banach spaces and of self-maps of cones that are non-expansive in Hilbert's, Thompson's or Riemannian metric, which have received a considerable attention, due in particular to applications in game theory [RS01, NS04], discrete event systems [Gun03], quadratic optimal control or filtering [Bou95], and non-linear Perron-Frobenius theory [Nus88|. Several

*INRIA Saclay \& Centre de Mathématiques Appliquées (CMAP), École Polytechnique, 91128 Palaiseau, France. email: Stephane.Gaubert@inria.fr, fax: +33169334646

†Université Paris-Dauphine, CEREMADE, Place du Maréchal De Lattre de Tassigny. 75775 Paris cedex 16, France. email: guillaumevigeral@gmail.com

This work was performed when the second author was with INRIA Saclay - Île-de-France and CMAP, École Polytechnique, being supported by the Digiteo project DIM08 PASO number 3389. The first author was partially supported by the Arpege programme of the French National Agency of Research (ANR), project "ASOPT", number ANR-08-SEGI-005. 
Denjoy-Wolff type results, either in the setting of metric spaces, or concerning the special case of cones have appeared, see in particular [Bea97, Kar01, AGLN06, Nus07, Lin07]. The reader may consult the monograph [RS05] for an overview of the field.

In this paper, we consider the linear escape rate

$$
\rho(T)=\lim _{k \rightarrow \infty} \frac{d\left(x, T^{k}(x)\right)}{k} .
$$

The latter always exists, by a standard subadditive argument. Our main result is the following maximin characterisation of the escape rate.

Theorem 1. Let $T$ be a non-expansive self-map of a complete metrically star shaped hemi-metric space $(X, d)$. Then,

$$
\inf _{y \in X} d(y, T(y))=\rho(T)=\max _{h} \inf _{x \in X} h(T(x))-h(x),
$$

where the maximum, which is attained, is taken over the set of Martin functions $h$ of $(X, d)$. If in addition the hemi-metric $d$ is bounded from below (in particular, if $d$ is a metric), and if $\rho(T)>0$, then any function attaining the maximum is a horofunction.

Let us explain the terminology. Hemi-metrics are analogous to metrics, but $d(x, y)$ and $d(y, x)$ may differ. Their definition is a variation of that of weak metrics in [PT09. The notion of metrically star shaped space is the object of Definition 5 below. It requires a distinguished family of geodesics with a common origin to satisfy a convexity inequality. Hence, it is a mild form of Busemann's classical non-positive curvature condition [Pap05]. Martin functions and horofunctions are special Lipschitz functions of constant 1 which arise when compactifying the hemi-metric space $(X, d)$. The set of horofunctions (the horoboundary) provides an abstract boundary of $X$. See $\$ 2.3$ for details.

Theorem 1 is inspired by the classical Collatz-Wielandt characterisation of the Perron root $\rho(M)$ of a $n \times n$ non-negative matrix $M$. The latter shows that

$$
\inf _{y \in \operatorname{int} \mathbb{R}_{+}^{n}} \max _{1 \leqslant i \leqslant n} \frac{(M y)_{i}}{y_{i}}=\rho(M)=\max _{\substack{u \in \mathbb{R}_{+}^{n} \\ u \neq 0}} \min _{\substack{1 \leqslant i \leqslant n \\ u_{i} \neq 0}} \frac{(M u)_{i}}{u_{i}} .
$$

Here, $\mathbb{R}_{+}$denotes the set of real non-negative numbers, and so $\mathbb{R}_{+}^{n}$ is the standard positive cone. Nussbaum showed in [Nus86] that the latter formula holds more generally when the map $y \mapsto M y$ is replaced by a non-linear continuous order-preserving $T$ of the standard positive cone. When $T$ preserves the interior of this cone, it is non-expansive in the (reverse) Funk hemi-metric [PT09,Wal08] defined on this interior by

$$
\operatorname{RFunk}(x, y):=\log \sup _{1 \leqslant i \leqslant n} \frac{y_{i}}{x_{i}} .
$$

Thus,

$$
\operatorname{RFunk}(T(x), T(y)) \leqslant \operatorname{RFunk}(x, y), \quad \forall x, y \in \operatorname{int} \mathbb{R}_{+}^{n} .
$$

The Collatz-Wielandt formula and its non-linear extension in [Nus86 will be shown to be special instances of the maximin formula 1.1 . 
Theorem 1 is also motivated by the case of a Banach space $X$. Then, one may consider the limit

$$
\lim _{k \rightarrow \infty} T^{k}(x) / k
$$

For instance, in the setting of zero-sum games, $T$ is the dynamic programming or Shapley operator [Sha53] (which acts on a Banach space of continuous functions equipped with the sup-norm), and the latter expression represents the limit of the mean payoff per time unit as a function of the initial position, when the horizon of the game tends to infinity, see $\$ 3.3$ for more background.

A theorem of Pazy Paz71 shows that the limit 1.3 does exist if $X$ is a Hilbert space; further improvements, under suitable strict convexity assumptions were done by Reich Rei73] and then by Kohlberg and Neyman [KN81]: the limit exists in the weak (resp. strong) topology if $X$ is reflexive and strictly convex (resp. if the norm of the dual space $X^{\star}$ is Fréchet differentiable). Without strict convexity, the limit may not exist, even when $X$ is finite dimensional, however, a general result of Kohlberg and Neyman KN81 (Corollary 20 below) shows that there is always a linear form $\varphi \in X^{\star}$ of norm 1 such that

$$
\varphi\left(T^{k}(x)\right) \geqslant \varphi(x)+k \bar{\rho}(T)
$$

holds for all $k \in \mathbb{N}$, where

$$
\bar{\rho}(T):=\inf _{y \in X} d(y, T(y))
$$

is precisely the term at the left-hand side of (1.1). The theorem of Kohlberg and Neyman may also be thought of as a special case of Theorem 1. Indeed, any horofunction $h$ attaining the maximum in (1.1) satisfies

$$
h\left(T^{k}(x)\right) \geqslant h(x)+k \bar{\rho}(T), \forall x \in X
$$

for all $k \in \mathbb{N}$. We shall see that the Kohlberg-Neyman theorem follows readily from this result. We shall also recover as corollaries a generalisation of a result of Gaubert and Gunawardena GG04 (Corollary 23) concerning Shapley operators, as well as a refinement of a result of Gunawardena and Walsh GW03 valid in symmetric cones, Corollary 32 below.

Theorem 1 turns out to be related to a Denjoy-Wolff type theorem of Karlsson [Kar01], Theorem 25 below. Karlsson showed that an inequality similar to (1.4) holds. He used a general subadditivity argument, which does not require any non-positive curvature condition. The statement of [Kar01] assumes the metric space to be proper, i.e., closed balls to be compact, but this assumption can be relaxed by defining horofunctions with respect to the topology of pointwise convergence as we do here. However, there are essential discrepancies between (1.4) and the result of |Kar01|: the term $\bar{\rho}(T)$ is replaced there by the linear escape rate $\rho(T)$, and the horofunction $h$ depends on the choice of the point $x$, whereas it does not in (1.4). We shall see that without a non-positive curvature condition, the formula (1.4) may not hold (we give an example in which $\bar{\rho}(T)>\rho(T)$ and $h$ necessarily depends on $x$ ). Hence, the maximin Theorem 1 holds only under more restrictive circumstances than the Denjoy-Wolff type result of Karlsson. By comparison, 
the interest of the more special Theorem 1 lies in its strong duality nature: it allows one to certify that $\rho(T)<\alpha$, or dually, that $\rho(T)>\beta$, by exhibiting an element $y \in X$ such that $d(y, T(y))<\alpha$, or dually a Martin function $h$ such that $\inf _{x \in X} h(T(x))-h(x)>\beta$. It should be noted in this respect that when $X$ is a Banach space with a polyhedral norm, the horoboundary admits a simple effective description, see [KMN06, Wal07, AGW09].

\section{Definitions and preliminary results}

\subsection{Metrically star-shaped spaces}

Definition 2. We say that $\delta: X \times X \rightarrow \mathbb{R}$ is a hemi-metric on a set $X$ if the two following conditions are satisfied for all $(x, y, z) \in X^{3}$ :

a) $\delta(x, z) \leqslant \delta(x, y)+\delta(y, z)$

b) $\delta(x, y)=\delta(y, x)=0$ if and only if $x=y$.

We then say that $(X, \delta)$ is a hemi-metric space.

Notice that a hemi-metric is generally not a metric, since it is neither symmetric nor non-negative. This definition is closely related to the one of a weak metric in $|\overline{\mathrm{PT} 09}|$, in which $\delta$ is required to be non-negative. The last condition of the definition corresponds to the weak separation condition in the latter reference. We allow $\delta$ to take negative values in order to deal with order-preserving positively homogeneous self-maps of cones (Section 4).

To any hemi-metric, one can canonically associate a metric by the following lemma.

Lemma 3. For any hemi-metric $\delta$, the function $d(x, y)=\max (\delta(x, y), \delta(y, x))$ is a metric on $X$.

Proof. Verifying that $d$ is symmetric and satisfies the triangular inequality is easy. The positivity of $d$ comes from the fact that for all $x$ and $y, 0=\delta(x, x) \leqslant \delta(x, y)+\delta(y, x)$. Finally, if $d(x, y)=0$ then both $\delta(x, y)$ and $\delta(y, x)$ are non-positive so by the same argument they are both null, thus $x=y$.

In the sequel, $X$ is equipped with the topology induced by the metric $d$. We shall say that $(X, \delta)$ is complete when the associated metric space $(X, d)$ is complete.

Definition 4. A geodesic joining a point $x \in X$ to a point $y \in X$ is a map $\gamma:[0,1] \rightarrow X$ such that $\gamma(0)=x, \gamma(1)=y$, and such that for all $0 \leqslant s \leqslant t \leqslant 1$,

$$
\delta(\gamma(s), \gamma(t))=(t-s) \delta(x, y) .
$$

Definition 5. We say that $(X, \delta)$ is metrically star-shaped with centre $x^{\circ}$ if there exists a family of geodesics $\left\{\gamma_{y}\right\}_{y \in X}$, such that $\gamma_{y}$ joins the centre $x^{\circ}$ to the point $y$, and such that the following inequality is satisfied for every $(y, z) \in X^{2}$ and $s \in[0,1]$ :

$$
\delta\left(\gamma_{y}(s), \gamma_{z}(s)\right) \leqslant s \delta(y, z)
$$

If the hemi-metric $\delta$ is not a metric, we also require that for any $y$, the quantity $\delta\left(y, \gamma_{y}(s)\right)$ tends to 0 as $s$ goes to 1 . 
The condition (2.1) is a form of metric convexity [Pap05]. In particular any Busemann space Pap05] is metrically star-shaped, but our definition is less demanding since we only require the inequality (2.1) to be satisfied for one specific choice of geodesics.

Example 6. Any Banach space $(X,\|\cdot\|)$ is metrically star-shaped with respect to any centre $x^{\circ}$ : it suffices to take the straight lines as geodesics, i.e., for any choice of centre $x^{\circ}$, the choice of $\gamma_{y}(s)=x^{\circ}+s\left(y-x^{\circ}\right)$ yields a metrically star shaped space. Notice that some Banach spaces are not Busemann spaces [Pap05.

The next example relies on the following notion.

Definition 7. A map $p$ from a Banach space $(X,\|\cdot\|)$ to $\mathbb{R}$ is a hemi-norm if it can be written as

$$
p(z)=\sup _{\phi \in E} \phi(z)
$$

where $E$ is a bounded subset of the dual space $X^{\star}$, and

$$
p(z)=p(-z)=0 \Longrightarrow z=0
$$

A hemi-norm is always Lipschitz. It is easily verified that if $p$ is a hemi-norm, then $\delta(x, y):=p(y-x)$ is a hemi-metric. Note that Condition 2.3 holds if and only if the orthogonal set of $E$ is reduced to the zero vector. As in the case of Banach spaces, $(X, \delta)$ is metrically star shaped: it suffices to choose the straight lines as geodesics. We shall say that the hemi-norm is compatible with the Banach space $(X,\|\cdot\|)$ if $\|z\|=$ $\max (p(z), p(-z))$. Then, the metric $d$ obtained from $\delta$ is the one associated to the norm of $X$, and in particular, the hemi-metric space $(X, \delta)$ is complete.

Example 8. The norm of the Banach space $X$ is a special case of compatible hemi-norm, in which

$$
p(z)=\|z\|=\sup _{\phi \in B^{\star}} \psi(z)
$$

where $B^{\star}$ is the dual unit ball of $X^{\star}$. Then, by Bauer maximum principle AB06, Theorem 7.69], the supremum is attained at some extreme point of $B^{\star}$, so the set $E$ arising in the definition of the hemi-norm (see Equation 2.2 can be taken to be either $B^{\star}$ or the set of extreme points of $B^{\star}$.

Example 9. A useful example of hemi-norm is the following. Let $\Omega$ denote a compact topological space, let $X=\mathscr{C}(\Omega)$ denote the Banach space of continuous functions from $\Omega$ to $\mathbb{R}$, equipped with the sup-norm. Consider $\delta(x, y)=t(y-x)$, where $t$ denotes the "top" operator which gives the maximum of a function, i.e.,

$$
t(x)=\max _{\omega \in \Omega} x(\omega)
$$

The metric $d$ obtained from $\delta$ is the sup-norm. We can take for $E$ the set of evaluations functions $\left\{e_{\omega}, \omega \in \Omega\right\}$ with $e_{\omega}(x)=x(\omega)$.

Example 10. Another class of examples concern symmetric cones, which include cones of positive semidefinite matrices. We shall discuss them further in Section 4 . 


\subsection{Non-expansive mappings}

In this section we consider a metrically star-shaped space $(X, \delta)$ with centre $x^{\circ}$, as well as a map $T: X \rightarrow X$ that is non-expansive with respect to the hemi-metric $\delta$, meaning that for all $(x, y) \in X^{2}$,

$$
\delta(T(x), T(y)) \leqslant \delta(x, y)
$$

Definition 11. To each non-expansive mapping $T$, we associate the two following quantities:

$$
\begin{gathered}
\bar{\rho}(T)=\inf _{x \in X} \delta(x, T(x)), \\
\rho(T)=\lim _{k \rightarrow+\infty} \frac{\delta\left(x, T^{k}(x)\right)}{k}=\inf _{k \geqslant 1} \frac{\delta\left(x, T^{k}(x)\right)}{k} .
\end{gathered}
$$

Thus, the number $\rho(T)$ measures the linear escape rate of the orbits of $T$. It is well defined. Indeed, for any $x \in X$, since $T$ is non-expansive, the sequence $u_{k}=\delta\left(x, T^{k}(x)\right)$ is subadditive, meaning that $u_{k+l} \leqslant u_{k}+u_{l}$ for all $k, l \geqslant 1$, and so, a classical argument (see [Bow08, Lemma 1.18]) shows that the $\operatorname{limit}_{k \rightarrow+\infty} k^{-1} \delta\left(x, T^{k}(x)\right)$ does exist and is equal to $\inf _{k \geqslant 1} k^{-1} \delta\left(x, T^{k}(x)\right)$. Moreover, since

$$
\begin{aligned}
\delta\left(x, T^{k}(x)\right) & \leqslant \delta(x, y)+\delta\left(y, T^{k}(y)\right)+\delta\left(T^{k}(y), T^{k}(x)\right) \\
& \leqslant \delta(x, y)+\delta(y, x)+\delta\left(y, T^{k}(y)\right)
\end{aligned}
$$

the limit is independent of the choice of $x \in X$.

Lemma 12. The following inequality is satisfied for any non-expansive mapping T:

$$
\rho(T) \leqslant \bar{\rho}(T) .
$$

Proof. Observe that for any $x \in X$ and $k \geqslant 1$,

$$
\delta\left(x, T^{k}(x)\right) \leqslant \sum_{l=0}^{k-1} \delta\left(T^{l}(x), T^{l+1}(x)\right) \leqslant k \delta(x, T(x))
$$

and take the infimum on both $k$ and $x$.

\subsection{The horofunction boundary}

The horofunction boundary of a metric space was defined by Gromov Gro81]. See also [BGS85], Bal95, Ch. II] and [Rie02]. In fact, the same construction can be performed with a hemi-metric. The details can be found in [AGW09], in which a horofunction-like boundary is defined for discrete optimal control problems, by analogy with the Martin compactification arising in the theory of Markov processes. See also [IM07].

Let us fix an arbitrary point $\bar{x} \in X$ (the basepoint). We define a map $i$ from $X$ to the set functions from $X$ to $\mathbb{R}$ by associating to any $x \in X$ the following function $i(x)$ :

$$
i(x): y \rightarrow[i(x)](y):=\delta(\bar{x}, x)-\delta(y, x) .
$$


Using the triangular inequality, we get that, for all $x, y \in X$,

$$
-\delta(y, \bar{x}) \leqslant[i(x)](y) \leqslant \delta(\bar{x}, y)
$$

Hence, the set $i(X):=\{i(x) \mid x \in X\}$ can be identified to a subset of the product space $\prod_{y \in X}[-\delta(y, \bar{x}), \delta(\bar{x}, y)] \subset \mathbb{R}^{X}$. By Tychonoff's theorem, the later space is compact for the product topology. Hence, the closure of $i(X)$ in the topology of pointwise convergence (which is the same as the product topology) is compact. We denote by $\mathscr{M}$ this closure. The elements of the boundary $\mathscr{H}:=\mathscr{M} \backslash i(X)$ are called horofunctions. We will call Martin functions the elements of $\mathscr{M}$ (the Martin space). Note that the choice of the basepoint is irrelevant (changing the basepoint translates all the Martin functions by the same constant).

Remark 13. The term of horofunction is sometimes used in a more restrictive sense, to denote what is also called a Busemann function, which is a horofunction obtained as the limit of a family of functions $\left(i\left(x_{s}\right)\right)_{s \geqslant 0}$ taken along an "infinite geodesic" $\left(x_{s}\right)_{s \geqslant 0}$, see [Rie02]. Note also the sign in the definition of $i(x)$ (which implies, in the Banach space case, that the map $i(x)$ is concave). This sign is chosen consistently with potential theory (the opposite of the distance is the analogue of the Martin kernel). In the metric geometry literature, the opposite choice is most often made.

Remark 14. The horofunctions are often defined as the closure of $i(X)$ in the topology of uniform convergence on bounded sets; indeed, the injection $x \mapsto i(x)$ is continuous for this topology, and this injection is an embedding if $X$ is a complete geodesic space Bal95. Ch. II]. It can be checked that every map $i(x)$ is Lipschitz of constant 1 with respect to the metric $d$, and so, by the Ascoli-Arzela theorem, the closure of $i(X)$ in this sense is compact as soon as $X$ is proper (meaning that every closed ball is compact). In the present work, we do not require $X$ to be proper, but define rather $\mathscr{M}$ as the closure of $i(X)$ in the topology of pointwise convergence, so that $\mathscr{M}$ is always compact. Note however that the injection $x \mapsto i(x)$ may not be an embedding from $X$ to $\mathscr{M}$ (the topology on $\mathscr{M}$ is too weak in general, so the inverse of the map $x \mapsto i(x)$ may not be continuous).

Remark 15. The topology of the Martin space is metrisable as soon as $X$ is a countable union of compact sets, see for instance AGW09, Remark 7.10]. Then, every horofunction is the limit of a sequence of functions $i\left(x_{m}\right)$, where $x_{m}$ is a sequence of elements of $X$.

\section{The main result and some of its consequences}

\subsection{The main result}

We shall derive Theorem 1 from the following result.

Theorem 16. Let $(X, \delta)$ be a complete metrically star-shaped hemi-metric space, and let $T: X \rightarrow X$ be non-expansive. Then there exists a Martin function $h \in \mathscr{M}$ such that for all $x \in X$,

$$
h(T(x)) \geqslant h(x)+\bar{\rho}(T) .
$$

Moreover, if $\delta$ is bounded from below (in particular, if $\delta$ is a metric) and if $\bar{\rho}(T)>0$, then $h$ is necessarily a horofunction. 
Proof. Let $x^{\circ}$ and $\left\{\gamma_{y}\right\}_{y \in X}$ as in Definition 5 , for any $\alpha \in\left[0,1\right.$ [denote by $r^{\alpha}: X \rightarrow X$ the function $y \rightarrow \gamma_{y}(\alpha)$ and recall that $r^{\alpha}$ is $\alpha$-contracting by definition. By completeness, we can thus define for any $\alpha$ the point $y_{\alpha} \in X$ as the only solution of the fixed point equation

$$
T\left(r^{\alpha}\left(y_{\alpha}\right)\right)=y_{\alpha}
$$

Then for any $x \in X$,

$$
\begin{aligned}
\delta\left(x, y_{\alpha}\right)-\delta\left(T(x), y_{\alpha}\right) & =\delta\left(x, y_{\alpha}\right)-\delta\left(T(x), T\left(r^{\alpha}\left(y_{\alpha}\right)\right)\right) \\
& \geqslant \delta\left(x, y_{\alpha}\right)-\delta\left(x, r^{\alpha}\left(y_{\alpha}\right)\right) \quad \text { (by non-expansiveness) } \\
& \geqslant \delta\left(x, y_{\alpha}\right)-\delta\left(x, r^{\alpha}(x)\right)-\delta\left(r^{\alpha}(x), r^{\alpha}\left(y_{\alpha}\right)\right) \\
& \geqslant(1-\alpha) \delta\left(x, y_{\alpha}\right)-\delta\left(x, r^{\alpha}(x)\right) \quad\left(\text { since } r^{\alpha} \text { is an } \alpha\right. \text {-contraction) } \\
& \geqslant(1-\alpha) \delta\left(x^{\circ}, y_{\alpha}\right)-(1-\alpha) \delta\left(x^{\circ}, x\right)-\delta\left(x, r^{\alpha}(x)\right) \\
& =\delta\left(r^{\alpha}\left(y_{\alpha}\right), y_{\alpha}\right)-(1-\alpha) \delta\left(x^{\circ}, x\right)-\delta\left(x, r^{\alpha}(x)\right) \\
& \geqslant \delta\left(T\left(r^{\alpha}\left(y_{\alpha}\right), T\left(y_{\alpha}\right)\right)\right)-(1-\alpha) \delta\left(x^{\circ}, x\right)-\delta\left(x, r^{\alpha}(x)\right) \\
& \left.=\delta\left(y_{\alpha}, T\left(y_{\alpha}\right)\right)\right)-(1-\alpha) \delta\left(x^{\circ}, x\right)-\delta\left(x, r^{\alpha}(x)\right) \\
& \geqslant \bar{\rho}(T)-(1-\alpha) \delta\left(x^{\circ}, x\right)-\delta\left(x, r^{\alpha}(x)\right) .
\end{aligned}
$$

Since the space $\mathscr{M}$ is compact, the family of functions $\left(i\left(y_{\alpha}\right)\right)_{0<\alpha<1}$ admits a limit point $h \in \mathscr{M}$ as $\alpha$ tends to 1 . Passing to the limit in the previous inequality, and using the additional assumption in Definition 5, we deduce that $-h(x)+h(T(x)) \geqslant \bar{\rho}(T)$.

Assume now by contradiction that $\delta$ is bounded from below, that $\bar{\rho}(T)>0$, and that $h=i(z)$ for some $z \in X$. Then, we deduce from $h(T(x)) \geqslant h(x)+\bar{\rho}(T)$ and $h(y)=-\delta(y, z)+\delta(b, z)$ that

$$
-\delta\left(T^{k}(x), z\right)+\delta(x, z) \geqslant k \bar{\rho}(T)
$$

The right-hand side of this expression tends to $+\infty$ as $k$ tends to infinity, but the left-hand side of this expression is bounded above independently of $k$, since $\delta\left(T^{k}(x), z\right)$ is bounded from below. This is impossible, hence, $h \in \mathscr{H}$ is a horofunction. (More generally, the same argument shows that for any net of functions $\left(i\left(x_{\beta}\right)\right)_{\beta \in B}$ converging to $h$, the net $\left(x_{\beta}\right)_{\beta \in B}$ cannot have a bounded subnet.)

We obtain as immediate consequences the following corollaries:

Corollary 17. Let $X, \delta$ and $T$ be as in Theorem 16. Then, there exists a Martin function $h \in \mathscr{M}$ such that for all $x \in X$,

$$
h\left(T^{k}(x)\right) \geqslant h(x)+k \bar{\rho}(T) .
$$

Moreover, if $\delta$ is bounded from below and if $\bar{\rho}(T)>0$, then $h$ is necessarily a horofunction.

Corollary 18. If the assumptions of Theorem 16 are satisfied, then $\bar{\rho}(T)=\rho(T)$. 
Proof. We deduce from (3.1) that

$$
\bar{\rho}(T) \leqslant \frac{1}{k}\left(h\left(T^{k}(x)\right)-h(x)\right) \leqslant \frac{\delta\left(x, T^{k}(x)\right)}{k}
$$

which yields $\bar{\rho}(T) \leqslant \rho(T)$. Then, the result follows by Lemma 12 .

Theorem 1 follows readily by combining Theorem 16 and Corollary 18 .

\subsection{The Kohlberg-Neyman theorem revisited}

We now apply Theorem 16 to non-expansive self-maps of a Banach space $X$, and more generally, to those maps that are non-expansive in a compatible hemi-norm, as defined in Section 2.1.

Theorem 19. Let $(X,\|\cdot\|)$ be a Banach space, and let

$$
p(z)=\sup _{\phi \in E} \phi(z)
$$

be a compatible hemi-norm, where $E$ is a bounded subset of the dual space $X^{\star}$. Let $T$ be non-expansive for $\delta$. Then, for every $x \in X$, there exists a linear form $\phi$ in the weak-star closure of $E$ such that for every $k \in \mathbb{N}$,

$$
\phi\left(T^{k}(x)\right) \geqslant \phi(x)+k \bar{\rho}(T) .
$$

Proof. Let clo $E$ denote the closure of $E$ in the weak-star topology. Observe that, for all $u \in X$,

$$
p(u)=\sup _{\phi \in \operatorname{clo} E} \phi(u)
$$

Since $E$ is bounded, clo $E$ is weak-star compact, and so, the map $\phi \mapsto \phi(u)$ attains its maximum on clo $E$.

Fix now $x$ and $y$ in $X$. It follows from the previous discussion that for all $z \in X$,

$$
i(y)(z)-i(y)(x)=p(y-x)-p(y-z)=\phi(y-x)-p(y-z)
$$

for some $\phi \in \operatorname{clo} E$, independent of $z$, and so

$$
i(y)(z)-i(y)(x) \leqslant \phi(y-x)-\phi(y-z)=\phi(z-x) .
$$

In other words, $\phi$ is in the super-differential of $i(y)$ at point $x$.

Let $h$ be the Martin function that appears in Corollary 17 $h=\lim _{D} i\left(y_{d}\right)$ for some net $\left(y_{d}\right)_{d \in D}$. By the previous observation, for every $d \in D$ we can find an element $\phi_{d} \in$ clo $E$ such that for every $k \in \mathbb{N}$,

$$
i\left(y_{d}\right)\left(T^{k}(x)\right)-i\left(y_{d}\right)(x) \leqslant \phi_{d}\left(T^{k}(x)-x\right) .
$$

Taking the limit along some subnet, we can find a linear form $\phi$ in clo $E$ such that for every $k \in \mathbb{N}$,

$$
h\left(T^{k}(x)\right)-h(x) \leqslant \phi\left(T^{k}(x)-x\right) .
$$

Since by Corollary $17 h\left(T^{k}(x)\right)-h(x) \geqslant k \bar{\rho}(T)$, the theorem is established. 
We now recover the following result.

Corollary 20 (Kohlberg and Neyman |KN81, Ney03|). Let $(X,\|\cdot\|)$ be a Banach space, let $T: X \rightarrow X$ be non-expansive and assume that $\bar{\rho}(T)>0$. Then for any $x \in X$, there exists a continuous linear form $\phi$ of norm one, such that

$$
\phi\left(T^{k}(x)\right) \geqslant \phi(x)+k \bar{\rho}(T)
$$

for all $k \in \mathbb{N}$. Moreover, $\phi$ can be taken in the weak-star closure of the set of extreme points of the dual unit ball.

Proof. We apply Theorem 19, taking $E$ to be the set of extreme points of the dual unit ball. This gives a $\phi$ in the weak-star closure of $E$ satisfying 3.2 . In particular $\|\phi\|_{\star} \leqslant 1$. Moreover, $\bar{\rho}(T) \leqslant \lim _{k \rightarrow \infty} k^{-1} \phi\left(T^{k}(x)-x\right) \leqslant\|\phi\|_{\star} \rho(T)$, and since $\rho(T)=\bar{\rho}(T)>0$, $1 \leqslant\|\phi\|_{\star}$, which shows that $\|\phi\|_{\star}=1$.

Actually, the assumption that $\bar{\rho}(T)>0$ can be dispensed with in the finite dimension case:

Corollary 21. Let $(X,\|\cdot\|)$ be a finite dimensional Banach space, and let $T: X \rightarrow X$ be non-expansive. Then for any $x \in X$, there exists a linear form $\phi$ of norm one, in the closure of the set of extreme points of the unit ball, such that

$$
\phi\left(T^{k}(x)\right) \geqslant \phi(x)+k \bar{\rho}(T)
$$

for all $k \in \mathbb{N}$.

Proof. The proof is similar to the one of Corollary 20. We still take $E$ as the set of extreme points of the dual unit ball, which belongs to the dual unit sphere, and we conclude since the unit sphere of a finite dimensional Banach space is closed.

Remark 22. The case in which $\rho(T)=0$, in finite dimension, has been studied in particular by Lins in [Lin09|, who proved that either $T$ has a fixed point or there exists a linear form $\varphi$ of norm 1 such that $\varphi\left(T^{k}(x)\right) \rightarrow+\infty$ for all $x \in X$. This result is established by showing first that in the latter case, there is a horofunction $h$ such that $h\left(T^{k}(x)\right) \rightarrow+\infty$ as $k$ tends to $\infty$ (modulo a change of sign convention for horofunctions). As noted in Remark 3.1 of [Lin09], the construction of the linear form $\varphi$ from the horofunction $h$ relies essentially on a sub-differentiability argument. An argument of the same nature is used here to derive Corollary 21 from Theorem 16. Note however that Corollary 21 and the existence of the horofunction in [Lin09] are independent results (none of them can be recovered from the other one).

\subsection{Application to Shapley operators}

In this section $\Omega$ is a compact topological space, and $X=\mathscr{C}(\Omega)$ is the Banach space of continuous functions from $\Omega$ to $\mathbb{R}$, endowed with the sup norm. We will be interested in functions $T: X \rightarrow X$ that are order-preserving, meaning that

$$
x \leqslant y \Longrightarrow T(x) \leqslant T(y)
$$


where $\leqslant$ denotes the canonical partial order on functions. We shall say that $T$ commutes with the addition of a constant if

$$
T(\lambda+x)=\lambda+T(x), \quad \forall \lambda \in \mathbb{R},
$$

where $\lambda+x$ denotes the function $\omega \mapsto \lambda+x(\omega)$.

As in Example 9, we set $t(x):=\max _{\omega \in \Omega} x(\omega)$ and consider $\delta(x, y)=t(y-x)$. Recall that $\delta$ is a hemi-metric compatible with the sup norm and that $(X, \delta)$ is metrically starshaped.

It is easy to see that $T$ is order-preserving and commutes with the addition of a constant if and only if

$$
t(T(x)-T(y)) \leqslant t(x-y)
$$

for all $x$ and $y$, meaning that $T$ is non-expansive for $\delta$ (see in particular [GK95], or [LS05]).

For this special hemi-metric $\delta$, we denote by $\bar{\rho}_{+}(T)$ and $\rho_{+}(T)$ the quantities defined in (2.4) and (2.5). Theorem 16 and Corollary 18 imply that

$$
\rho_{+}(T)=\lim _{k \rightarrow+\infty} \max _{\omega \in \Omega} \frac{T^{k}(x)(\omega)-x(\omega)}{k}=\bar{\rho}_{+}(T)=\inf _{y \in X} \max _{\omega \in \Omega}(T(y)(\omega)-y(\omega))
$$

for all $x$ in $X$.

We next derive from Theorem 19 the following generalisation of a result established in $\overline{\text { GG04 }}$ when $\Omega$ is finite.

Corollary 23. Let $\Omega$ be a compact topological space, and let $X=\mathscr{C}(\Omega)$ be the Banach space of continuous functions from $\Omega$ to $\mathbb{R}$, endowed with the sup norm. Suppose that $T: X \rightarrow X$ is order-preserving and commutes with the addition of a constant. Then for every $x \in X$, there exists $\omega_{+} \in \Omega$ such that,

$$
T^{k}(x)\left(\omega_{+}\right) \geqslant x\left(\omega_{+}\right)+k \bar{\rho}_{+}(T)
$$

for all $k \in \mathbb{N}$.

Proof. We apply Theorem 19 to the hemi-norm $p(z)=t(z)$, taking for $E$ the set of evaluation functions $e_{\omega}, \omega \in \Omega$. Since $\Omega$ is compact, the set $E$ is weak-star closed, so that the linear form $\phi$ in Theorem 19 can be written as $\phi=e_{\omega_{+}}$for some $\omega_{+} \in E$.

Order-preserving maps $T$ commuting with the addition of a constant arise as Shapley operators in game theory. Suppose now that $\Omega, A$ and $B$ are three compact metric spaces, that $g$ is a continuous function from $A \times B \times \Omega$ to $\mathbb{R}$, and that $q: A \times B \times \Omega \rightarrow \Delta(\Omega)$ is continuous, where $\Delta(\Omega)$, the set of probabilities on $\Omega$, is endowed with the weak topology. Then the Shapley operator of the two-player, zero-sum stochastic game with state space $\Omega$, action sets $A$ and $B$, payoff $g$, and transition probability $q$, is defined as

$$
\begin{aligned}
T(x)(\omega) & =\sup _{\mu \in \Delta(A)} \inf _{\nu \in \Delta(B)}\left\{\int_{A \times B}\left[g(a, b, \omega)+\int_{\Omega} q\left(d w^{\prime} \mid a, b, \omega\right) x\left(\omega^{\prime}\right)\right] \mu(d a) \nu(d b)\right\} \\
& =\inf _{\nu \in \Delta(B)} \sup _{\mu \in \Delta(A)}\left\{\int_{A \times B}\left[g(a, b, \omega)+\int_{\Omega} q\left(d w^{\prime} \mid a, b, \omega\right) x\left(\omega^{\prime}\right)\right] \mu(d a) \nu(d b)\right\} .
\end{aligned}
$$


The map $T$ is order-preserving and commutes with the addition of a constant. It is shown in |MSZ94, Now03| that it preserves $\mathscr{C}(\Omega)$. Then, $\frac{T^{k}(0)(\omega)}{k}$ represents the average reward per time unit if both players play optimally in the $k$-stage game and if the starting state is $\omega$. Thus, Corollary 23 shows that there is an initial state $\omega_{+}$which is the best for the maximising player in the long term. In particular $\frac{T^{k}(0)\left(\omega_{+}\right)}{k}$ converges as $k$ goes to infinity.

Remark 24. To every result concerning the hemi-norm $t$ corresponds a dual result concerning the hemi-norm $b(x):=-t(-x)=\min _{\omega \in \Omega} x(\omega)$. In particular,

$$
\rho_{-}(T):=\lim _{k \rightarrow+\infty} \min _{\omega \in \Omega} \frac{T^{k}(x)(\omega)-x(\omega)}{k}=\bar{\rho}_{-}(T):=\sup _{y \in X} \min _{\omega \in \Omega}(T(y)(\omega)-y(\omega))
$$

Moreover, there exists $\omega_{-} \in \Omega$ satisfying

$$
T^{k}(x)\left(\omega_{-}\right) \leqslant x\left(\omega_{-}\right)+k \bar{\rho}_{-}(T)
$$

for all $k \in \mathbb{N}$. This is readily deduced by applying Corollary 23 to the map $x \rightarrow-T(-x)$.

\subsection{Comparison with a theorem of Karlsson}

Theorem 16 should be compared with the following result. (Recall that a metric space is proper if every closed ball is compact.)

Theorem 25 (Karlsson |Kar01, Th. 3.3]). If $T$ is a non-expansive self-map of a proper metric space $(X, \delta)$, then, for all $x \in X$, there exists a horofunction $h$ (depending on $x$ ), such that

$$
h\left(T^{k}(x)\right) \geqslant h(x)+k \rho(T)
$$

holds for all $k \geqslant 1$.

Note first two differences in the statements of Theorem 16 and of Karlsson's theorem: we require a metric convexity assumption whereas Karlsson does not; Karlsson makes the general assumption that the metric space is proper whereas we do not. Actually, the compactness issue appears to be a mere technicality: it can be checked that the proof of Theorem 25 remains valid even if $X$ is not proper if one defines horofunctions with respect to the topology of pointwise convergence as we do here (see Remark 14).

A key difference however lies in the conclusions of both results. In Theorem 16, horofunctions are independent of the choice of $x$, whereas in Karlsson's result, the horofunction does depend on $x$. Note also that inequalities involving $\bar{\rho}(T)$ as in Theorem 16 and Corollary 17 are stronger than their counterparts involving $\rho(T)$ since $\bar{\rho}(T) \geqslant \rho(T)$. This is illustrated in Example 26 below, in which we consider a non-star-shaped space for which Theorem 25 applies but not Theorem 16. This example shows that the assumptions of Theorem 16 cannot be weakened : it is primordial that the space $X$ is metrically starshaped. Hence, Theorem 16 and Karlsson's result are incomparable: the latter holds under more general circumstances, but the former yields a stronger conclusion.

Example 26 . Let $\mathbb{T}:=\mathbb{R}(\bmod 1)$ be the torus with its canonical metric $d_{\mathbb{T}}$; and let $X=$ $\mathbb{T} \times \mathbb{R}$, with the metric

$$
d\left((x, t),\left(x^{\prime}, t^{\prime}\right)\right):=d_{\mathbb{T}}\left(x, x^{\prime}\right)+\left|t-t^{\prime}\right| .
$$


Let $\alpha \in\left(0, \frac{1}{2}\right) \backslash \mathbb{Q}$, and consider the mapping $T: X \rightarrow X$,

$$
T(x, t)=(x+\alpha, t+1)
$$

which is non-expansive for the metric $d$. It is straightforward to check that $\rho(T)=1$ but $\bar{\rho}(T)=1+\alpha$, so inequation (3.1) cannot be satisfied (otherwise one would have $\rho(T)=\bar{\rho}(T)$ by Corollary 18$)$.

In fact we prove that it is not even true that there exists a function $h \in \mathscr{M}$ such that for all $x$,

$$
h\left(T^{k}(x, t)\right) \geqslant h(x, t)+k \rho(T), \quad \forall k \in \mathbb{N} .
$$

Fix a basepoint $(\bar{x}, \bar{t})$ in $X$. Since the metric on $X=\mathbb{T} \times \mathbb{R}$ is the sum of the two metrics $\left(\mathbb{T}, d_{T}\right)$ and $(\mathbb{R},|\cdot|)$, we can write any Martin function $h$ of $X$ as the sum

$$
h(x, t)=h_{1}(x)+h_{2}(t)
$$

where $h_{1} \in$ is a Martin function of $\left(\mathbb{T}, d_{T}\right)$ and $h_{2}$ is a Martin function of $(\mathbb{R},|\cdot|)$, relatively to the basepoints $\bar{x}$ and $\bar{t}$, respectively (see Prop. 9.1 in [AGW09]). The only Martin functions on $\mathbb{T}$ are $h_{1}(x)=-d_{\mathbb{T}}(x, y)+d_{\mathbb{T}}(\bar{x}, y)$ with $y \in \mathbb{T}$. The Martin functions of $(\mathbb{R},|\cdot|)$ are either of the form $h_{2}(t)=-|t-s|+|\bar{t}-s|$, with $s \in \mathbb{R}$, or the affine functions $h_{2}(t)=t-\bar{t}$ and $h_{2}=-(t-\bar{t})$. We search a Martin function $h$ such that, for any $(x, t) \in X$,

$$
h\left(T^{k}(x, t)\right) \geqslant h(x, t)+k, \quad \forall k \in \mathbb{N}
$$

i.e.,

$$
h_{1}(x+k \alpha)+h_{2}(t+k) \geqslant h_{1}(x)+h_{2}(t)+k, \quad \forall k \in \mathbb{N} .
$$

Any Martin function $h_{1}$ is bounded. If $h_{2}$ is of the form $h_{2}(t)=-|t-s|+|\bar{t}-s|$ or $h_{2}(t)=-(t-\bar{t})$, then, for a fixed $(x, t)$, the left-hand side of (3.7) remains bounded from above as $k \rightarrow \infty$, whereas the right-hand side of (3.7) tends to infinity. The only possibility is thus $h_{2}(t)=t-\bar{t}$. In that case, (3.7) becomes

$$
h_{1}(x+k \alpha)-h_{1}(x) \geqslant 0 .
$$

Since the sequence $(x+k \alpha)_{k \in \mathbb{N}}$ is dense in $\mathbb{T}$, and since $h_{1}$ is continuous, we have that $h_{1}(x) \leqslant \inf _{y \in \mathbb{T}} h_{1}(y)$. This is true for any $x \in \mathbb{T}$, so $h_{1}$ is constant. But there are no constant Martin function of $\left(\mathbb{T}, d_{\mathbb{T}}\right)$.

Karlsson's result shows however that for any choice of $(x, t) \in X$, there exists a horofunction $h$ (depending on $(x, t))$ such that $(3.6)$ is satisfied.

\section{A Denjoy-Wolff type theorem for order-preserving homogeneous self-maps of symmetric cones}

\subsection{The reverse Funk metric}

In this section, we recall or establish preliminary results concerning cones. We consider a (closed, convex and pointed) cone $C$ in $\mathbb{R}^{n}$ with non-empty interior $X:=\operatorname{int} C$. We equip 
$C$ with the partial order defined by $x \leqslant y$ if $y-x \in C$. For all $x, y \in C \backslash\{0\}$, we set

$$
M(y / x):=\inf \{\lambda>0 \mid \lambda x \geqslant y\}, \quad m(y / x):=\sup \{\lambda>0 \mid \lambda x \leqslant y\} .
$$

Since $C$ is closed, as soon as the two latter sets are non-empty, their respective infimum and supremum are attained; in particular, $M(y / x)$ and $m(y / x)$ are finite and positive.

The (reverse) Funk hemi-metric on the interior of $C$ is defined by

$$
\operatorname{RFunk}(x, y):=\log M(y / x) .
$$

More generally, we shall use the notation $\operatorname{RFunk}(x, y)$ as soon as $x, y \in C \backslash\{0\}$ are such that $\mu x \geqslant y$ for some $\mu>0$. The terminology "Funk metric" is borrowed from PT09, Wal08; it refers to [Fun29]. The map $\delta:=$ RFunk is easily checked to be a hemi-metric on int $C$. Indeed: $\delta(x, y)$ is finite since $x$ is in the interior of $C$; the triangular inequality and the fact that $\delta(x, x)=0$ for any $x \in X$ are easy to verify since $C$ is pointed; the fact that $\delta(y, x)=\delta(y, x)=0 \Longrightarrow x=y$ follows from the fact that $C$ is closed and pointed. The term "reverse" is by opposition to the hemi-metric $(x, y) \mapsto \delta(y, x)$ which has a different horoboundary [Wal08.

The quantities $M(y / x)$ and $m(y / x)$ can be expressed in terms of the extreme rays of the dual cone $C^{\star}$. The latter is the set of continuous linear forms taking non-negative values on $C$. For each cone $K$, Extr $K$ denotes the set of representatives of the extreme rays of $K$ (i.e., the non-zero vectors belonging to these extreme rays).

Lemma 27. Let $x, y, z \in C \backslash\{0\}$ be such that $\mu x \geqslant y$ and $z \geqslant \nu x$ for some $\mu, \nu>0$. Then,

$$
\begin{aligned}
& M(y / x)=\sup _{\varphi \in C^{\star}, \varphi(x) \neq 0} \frac{\varphi(y)}{\varphi(x)}=\sup _{\varphi \in \operatorname{Extr} C^{\star}, \varphi(x) \neq 0} \frac{\varphi(y)}{\varphi(x)}, \\
& m(z / x)=\inf _{\varphi \in C^{\star}, \varphi(x) \neq 0} \frac{\varphi(z)}{\varphi(x)}=\inf _{\varphi \in \operatorname{Extr} C^{\star}, \varphi(x) \neq 0} \frac{\varphi(z)}{\varphi(x)} .
\end{aligned}
$$

Moreover, when $x \in \operatorname{int} C$, the latter suprema are attained, and the condition that $\varphi(x) \neq 0$ can be replaced by $\varphi \in C^{\star} \backslash\{0\}$. When $z \in \operatorname{int} C$, the latter infima are attained.

This result is somehow standard. We include the proof for the convenience of the reader.

Proof. Since $C=\left(C^{\star}\right)^{\star}$, the inequality $\lambda x \geqslant y$, i.e., $\lambda x-y \in C$, is equivalent to $\varphi(\lambda x-$ $y) \geqslant 0$, for all $\varphi \in C^{\star}$, from which the first equality in (4.1) follows.

Let now recall that if $u$ is in the interior of $C$, and if $\varphi \in C^{\star} \backslash\{0\}$, then, $\varphi(u)$ cannot vanish. Indeed, we can find a ball $B$ centred at 0 , such that $u+z \in C$ for all $z \in B$. If $\varphi(u)=0$, then, $0 \leqslant \varphi(u \pm z)= \pm \varphi(z)$ holds for all $z \in B$, and so $\varphi=0$.

Choose now a vector $u \in \operatorname{int} C$, and define $\Sigma_{u}:=\left\{\varphi \in C^{\star} \mid \varphi(u)=1\right\}$. Observe that $\Sigma_{u}$ is compact and convex, and that each extreme ray of $C$ can be identified to the unique extreme point of $\Sigma_{u}$ which generates this ray. Let $\bar{\lambda}$ denote the value of the last supremum in (4.1), so that the inequality $\bar{\lambda} \varphi(x) \geqslant \varphi(y)$ holds for all extreme points $\varphi$ of $\Sigma_{u}$. Since every element $\varphi$ of $\Sigma_{u}$ is a barycentre of extreme points of $\Sigma_{u}$, we deduce that 
the same inequality holds for all points $\varphi$ of $\Sigma_{u}$, which shows that $\bar{\lambda}$ coincides with the first supremum in (4.1).

The arguments to establish 4.2 are dual.

Finally, when $x \in \operatorname{int} C$, we consider the map $J: \Sigma_{x} \rightarrow \mathbb{R}, \varphi \mapsto \varphi(y) / \varphi(x)=\varphi(y)$. Since $J$ is linear, it attains its maximum at an extreme point of the compact convex set $\Sigma_{x}$, which implies that both suprema in (4.1) are attained. Similarly, when $z \in \operatorname{int} C$, we note that every $\varphi \in \Sigma_{z}$ maximising $\varphi(x)$ attains the first infimum in (4.2), and so, the second infimum is attained at an extreme point of $\Sigma_{z}$.

Walsh showed in Wal08 that the horoboundary of int $C$ in the (reverse) Funk hemimetric coincides with the space of rays contained in the Euclidean boundary of $C$.

Proposition 28 (|Wal08, Prop. 2.5]). Let $C$ be a closed convex pointed cone of nonempty interior in a finite dimensional Banach space, with basepoint $\bar{x}$. Then, any Martin function $h$ for the (reverse) RFunk hemi-metric corresponds to a vector $u \in C \backslash\{0\}$,

$$
h(x)=-\operatorname{RFunk}(x, u)+\operatorname{RFunk}(\bar{x}, u), \forall x \in \operatorname{int} C,
$$

and $h$ is a horofunction if and only if $u \in \partial C \backslash\{0\}$.

We shall also be interested in the following variant of the Funk hemi-metric, considered by Papadopoulos and Troyanov |PT09|:

$$
\operatorname{RFunk}^{+}(x, y):=\max (\operatorname{RFunk}(x, y), 0) .
$$

The map RFunk ${ }^{+}$is easily seen to be a hemi-metric. The following simple application of the arguments of [Wal08] characterises the boundary of the hemi-metric RFunk ${ }^{+}$.

Proposition 29. Let $C$ be a closed convex pointed cone of non-empty interior in a finite dimensional Banach space. Then, the Martin space for the RFunk ${ }^{+}$hemi-metric consists of the functions (4.3), with $u \in C \backslash\{0\}$, together with the functions

$$
h(x)=-\operatorname{RFunk}^{+}(x, u)+\operatorname{RFunk}^{+}(\bar{x}, u), \forall x \in \operatorname{int} C,
$$

with $u \in C$, and an element is a horofunction if and only if it is of the form (4.3), with $u \in C \backslash\{0\}$, or of the form (4.4) with $u \in \partial C$.

Proof. Any function $h$ of the Martin space is the pointwise limit of a sequence of functions $h_{m}: x \mapsto-\operatorname{RFunk}^{+}\left(x, z_{m}\right)+\operatorname{RFunk}^{+}\left(\bar{x}, z_{m}\right)$, where $\left(z_{m}\right)_{m \geqslant 1}$ is a sequence of elements of int $C$. If $\left\|z_{m}\right\| \rightarrow \infty$ as $m$ tends to infinity, then, for all $x \in \operatorname{int} C, \operatorname{RFunk}\left(x, z_{m}\right)$ also tends to infinity, and so $\operatorname{RFunk}^{+}\left(x, z_{m}\right)=\operatorname{RFunk}\left(x, z_{m}\right)$ for $m$ large enough. Then, the result of [Wal08 shows that $h=\lim _{m} h_{m}$ is of the form (4.3), for some $u \in C \backslash\{0\}$. It remains to examine the case in which $z_{m}$ contains a bounded subsequence. Then, possibly after replacing $z_{m}$ by a subsequence, we may assume that $z_{m}$ converges to a point $u \in C$. Using the fact established in [Wal08] that for $x \in \operatorname{int} C, \operatorname{RFunk}(x, y)$ is continuous in the second argument $y \in C$, we deduce that $h_{m}$ converges to the function (4.4). Finally, the characterisation of the horofunctions is straightforward. 
The metric $d_{T}$ associated to the Funk hemi-metric,

$$
d_{T}(x, y)=\max (\operatorname{RFunk}(x, y), \operatorname{RFunk}(y, x))
$$

is called the Thompson metric. We recall that for this metric, the map

$$
\gamma_{y}(s)=\frac{\beta^{s}-\alpha^{s}}{\beta \alpha^{s}-\alpha \beta^{s}+\beta^{s}-\alpha^{s}} x^{\circ}+\frac{\beta^{s}-\alpha^{s}}{\beta \alpha^{s}-\alpha \beta^{s}+\beta^{s}-\alpha^{s}} y
$$

with $\beta=\exp \left(\operatorname{RFunk}\left(x^{\circ}, y\right)\right)$ and $\alpha=\exp \left(-\operatorname{RFunk}\left(y, x^{\circ}\right)\right)$ is a (straight line) geodesic joining $x^{\circ}$ to $y$, see [Nus88]. However, as shown in [NW04], this choice of geodesics does not satisfy Busemann's non-positive curvature condition (even when $C=\mathbb{R}_{+}^{3}$ ). Actually, it is shown in [NW04 that only a weaker inequality is valid. The latter inequality is optimal (it is straightforward to find explicit counter examples to the convexity inequality).

However, when $C$ is the cone $S_{n}^{+}$of $n \times n$ positive semi-definite matrices, there is a different choice of geodesics between any pair of matrices $Z, Y$ in the interior of $C$, involving geometric means:

$$
\gamma_{Y}(s)=Z^{\frac{1}{2}}\left(Z^{-\frac{1}{2}} Y Z^{-\frac{1}{2}}\right)^{s} Z^{\frac{1}{2}}
$$

See in particular [Nus88, NW04]. The latter are indeed known to be geodesics in Thompson's and Hilbert's metric. Note that when $Z$ is the identity matrix, this geodesic seen with logarithmic glasses is a straight line: $\log \gamma_{Y}(s)=s \log Y$. The general form of the geodesic is obtained by considering the linear transformation $X \mapsto Z^{-1 / 2} X Z^{-1 / 2}$, which is an automorphism of the cone of positive definite matrices, isometric both in Thompson's and Hilbert's metric, and sending $Z$ to the identity. This construction actually makes sense more generally when $C$ is a symmetric cone [FK94], meaning that $C$ is self-dual $\left(C=C^{\star}\right)$, and that the group of automorphisms leaving invariant the interior of $C$ acts transitively on this interior. Symmetric cones include in particular the Lorentz cones, the cones of real or complex positive semidefinite matrices, and Cartesian products of such cones. It is shown in GW03 that the metric convexity property of Definition 5 holds for any symmetric cone, for the latter choice of geodesics, for any choice of the centre, both for the Thompson and Hilbert metric. A similar observation was made by Lawson and Lim LL07b. Actually, the arguments in [GW03 apply as well to the Funk and RFunk ${ }^{+}$ hemi-metrics. Hence, the present results apply to those self-maps of the interior of a symmetric cone that are non-expansive in any of the previously mentioned hemi-metrics.

Remark 30. When $C=S_{n}^{+}$is the cone of positive semi-definite matrices, when $x \in \operatorname{int} C$, and $y \in C \backslash\{0\}, M(y / x)$ coincides with the maximal eigenvalue of the symmetric matrix $v:=x^{-1 / 2} y x^{-1 / 2}$. Moreover, denoting by $w$ the (rank one) orthogonal projector on any eigenline corresponding to this eigenvalue, one can check that the linear form $\varphi(u):=$ $\operatorname{tr}\left(x^{-1 / 2} w x^{-1 / 2} u\right)$ attains the supremum in 4.1). When $x \in C \backslash\{0\}$, with $y \leqslant \mu x$ for some $\mu>0$, a maximising linear form $\varphi$ can be constructed in the same way, replacing $x^{-1 / 2}$ by the square root of the Moore-Penrose inverse of $x$. A dual argument allows one to construct a linear form attaining the infimum in (4.2). Note also that these constructions can be easily adapted to the case of a symmetric cone $C$. 
Remark 31. In the case of the cone of symmetric positive definite matrices, the Thompson, Hilbert, Funk, and RFunk ${ }^{+}$hemi-metrics all have the following form

$$
\delta_{\nu}(A, B):=\nu\left(\log \operatorname{Spec}\left(A^{-1} B\right)\right)=\nu\left(\log \lambda_{1}, \ldots, \log \lambda_{n}\right) \quad A, B \in \operatorname{int} S_{n}^{+},
$$

where $\nu$ is a symmetric function $\mathbb{R}^{n} \rightarrow \mathbb{R}$ (by symmetric, we mean that $\nu$ is invariant by permutation of its variables), $\operatorname{Spec}(M)$ denotes the sequence of eigenvalues of a matrix $M$, counted with multiplicities, so that $\lambda_{1}, \cdots, \lambda_{n}$ denote the eigenvalues of the matrix $A^{-1} B$ (which are real and positive since $A$ and $B$ are positive definite). The previously listed hemi-metrics are obtained by taking for $\nu$ the following maps, $\mathbb{R}^{n} \rightarrow \mathbb{R}$,

$$
\mu \mapsto \max _{1 \leqslant i \leqslant n}\left|\mu_{i}\right|, \quad \mu \mapsto \max _{1 \leqslant i, j \leqslant n}\left|\mu_{i}-\mu_{j}\right|, \quad \mu \mapsto \max _{1 \leqslant i \leqslant n} \mu_{i}, \quad \mu \mapsto \max \left(\max _{1 \leqslant i \leqslant n} \mu_{i}, 0\right)
$$

respectively. We may consider more generally any symmetric hemi-norm $\nu$. Then, the corresponding map $\delta_{\nu}$ can be checked to be a hemi-metric, which is of Finsler type [Nus94. Indeed, measuring the length of a vector $Y$ in the tangent space of $S_{n}^{+}$at point $X$ by the hemi-norm $\nu\left(\operatorname{Spec}\left(X^{-1} Y\right)\right)$, we get

$$
\delta_{\nu}(A, B)=\inf _{\beta} \int_{0}^{1} \nu\left(\operatorname{Spec}\left(\beta(s)^{-1} \dot{\beta}(s)\right) d s,\right.
$$

the infimum being taken over the set of curves $\beta$ from $[0,1]$ to the interior of the cone, such that $\beta(0)=A$ and $\beta(1)=B$. We recover as special cases of this construction the invariant Riemannian metric [Mos55] ( $\nu$ is the Euclidean norm), the $\log p$-Schatten metric [FF04 ( $\nu$ is the $\ell_{p}$ norm), as well as metrics arising from symmetric Gauge functions [Bha03] (the latter are symmetric hemi-norms that are invariant by a change of sign of each variable, and take positive values except at the origin). For any choice of symmetric hemi-metric $\nu$, the map (4.6) yields a family of geodesics for the hemi-metric $\delta_{\nu}$ joining a centre $Z$ to any matrix $Y$, for which int $S_{n}^{+}$is a metrically star shaped space. This can be proved along the lines of [Bha03], where the same property is established in the special case in which $\nu$ is a symmetric Gauge function. The reader may also consult ACS00, GW03, in which the significance of several matrix inequalities in terms of non-positive curvature is brought to light.

A number of non-linear maps, including discrete Riccati operators (Example 40 below), are known to be non-expansive in various metrics $\delta_{\nu}$, see [Bou95, LW94, LL08, LL07a].

\subsection{A Denjoy-Wolff type theorem}

We shall now consider specifically the case of order-preserving and positively homogeneous maps. A self-map $T$ of $C$ is order-preserving if $x \leqslant y \Longrightarrow T(x) \leqslant T(y)$. It is positively homogeneous (of degree one) if $T$ commutes with the product by a positive constant. These notions make sense more generally if $T$ is only defined on a subset of $C$ invariant by any such product. It is readily checked that a self-map $T$ of the interior of $C$ is orderpreserving and positively homogeneous if and only if it is non-expansive in the Funk metric. 
Corollary 32. Let $T$ be an order-preserving and positively homogeneous self-map of the interior of a symmetric cone $C$, and let

$$
\bar{\rho}(T):=\inf _{y \in \operatorname{int} C} \operatorname{RFunk}(y, T(y)) .
$$

Then, for all $x \in \operatorname{int} C$,

$$
\bar{\rho}(T)=\rho(T):=\lim _{k \rightarrow \infty} \frac{\operatorname{RFunk}\left(x, T^{k}(x)\right)}{k}
$$

and there exists an extreme ray of $C$, such that for any element $w$ in this ray,

$$
\log \left\langle w, T^{k}(x)\right\rangle \geqslant \log \langle w, x\rangle+k \bar{\rho}(T) .
$$

Here $\langle\cdot, \cdot\rangle$ denotes the scalar product of the Euclidean space in which $C$ is self-dual.

Proof. The map $T$ is non-expansive in the Funk metric. By Corollary 18, $\rho(T)=\bar{\rho}(T)$. Let $h$ be the Martin function appearing in Theorem 16 . Then, there exists a sequence of elements of int $C,\left(z_{m}\right)_{m \geqslant 1}$ such that $i\left(z_{m}\right)$ converges to $h$. Let us now fix $x \in \operatorname{int} C$. Since $C=C^{\star}$, any element $\varphi$ of an extreme ray of $C^{\star}$ can be written as $\varphi(y)=\langle w, y\rangle$ where $w$ is an element of an extreme ray of $C$. Then, it follows from Lemma 27 that for all $m$, there exists an element $w_{m} \in \operatorname{Extr} C$ such that

$$
\operatorname{RFunk}\left(x, z_{m}\right)=\log \frac{\left\langle w_{m}, z_{m}\right\rangle}{\left\langle w_{m}, x\right\rangle} .
$$

A ray of $C$ is known to be extreme if and only if it contains a primitive idempotent of the associated Jordan algebra [FK94, Prop. IV.3.2]. Moreover, the set of these primitive idempotents is compact (Exercise 5 of Chap. 4 in [FK94|). Since the previous property is invariant by a scaling of $w_{m}$, we may assume that each $w_{m}$ is a primitive idempotent, and so, possibly after extracting a subsequence, we may assume that $w_{m}$ converges to a primitive idempotent $w$, which therefore belongs to an extreme ray of $C$. For all $k \geqslant 0$, we get

$$
\begin{aligned}
\log \left\langle w, T^{k}(x)\right\rangle-\log \langle w, x\rangle & =\lim _{m} \log \left\langle w_{m}, T^{k}(x)\right\rangle-\log \left\langle w_{m}, x\right\rangle \\
& \geqslant \lim _{m}-\operatorname{RFunk}\left(T^{k}(x), z_{m}\right)+\operatorname{RFunk}\left(x, z_{m}\right) \\
& =h\left(T^{k}(x)\right)-h(x) \geqslant k \bar{\rho}(T)
\end{aligned}
$$

which concludes the proof.

We next point out a variant of Corollary 32 concerning order-preserving and subhomogeneous self-maps of the interior of a symmetric cone. Recall that sub-homogeneous means that for all $x \in \operatorname{int} C$ and $\lambda \geqslant 1, T(\lambda x) \leqslant \lambda T(x)$. Order-preserving and subhomogeneous maps are easily seen to be characterised by the following property

$$
\operatorname{RFunk}^{+}(T(x), T(y)) \leqslant \operatorname{RFunk}^{+}(x, y)
$$


Corollary 33. Let $T$ be an order-preserving and sub-homogeneous self-map of the interior of a symmetric cone $C$, and let

$$
\bar{\rho}(T):=\inf _{y \in \operatorname{int} C} \operatorname{RFunk}^{+}(y, T(y))
$$

Then, for all $x \in \operatorname{int} C$,

$$
\bar{\rho}(T)=\rho(T):=\lim _{k \rightarrow \infty} \frac{\operatorname{RFunk}^{+}\left(x, T^{k}(x)\right)}{k}
$$

and, if $\bar{\rho}(T)>0$, there exists an extreme ray of $C$, such that for any element $w$ in this ray,

$$
\log \left\langle w, T^{k}(x)\right\rangle \geqslant \log \langle w, x\rangle+k \bar{\rho}(T)
$$

Proof. Since the hemi-metric $\mathrm{RFunk}^{+}$is bounded from below, the observation made at the end of the proof of Theorem 16 shows that if $z_{m}$ is any sequence of points of int $C$ such that $i\left(z_{m}\right)$ converges to the Martin function $h$ in this theorem, then, the sequence $z_{m}$ must be such that $\left\|z_{m}\right\| \rightarrow \infty$. Then, it follows from the characterisation of the boundary of the RFunk ${ }^{+}$metric (proof of Proposition 29) that $h$, which cannot be of the form (4.4), is necessarily of the form (4.3). Therefore, we conclude as in the proof of Corollary 32.

Remark 34. Corollary 32 should be compared with a related result of Gunawardena and Walsh [GW03, Th. 1], in which the map $T$ is only required to be non-expansive in Thompson's metric. Then, it is shown that all the accumulation points of the sequence $\left(\log T^{k}(x)\right) / k$ belong to the same face of a ball which respect to the norm in the tangent space of $C$ at point $x$.

Remark 35. Lins established a Denjoy-Wolff theorem of a different nature in [Lin07], concerning self-maps of a polyhedral domain that are non-expansive in Hilbert's metric.

\subsection{The Collatz-Wielandt theorem}

We now show that the Collatz-Wielandt formula $(1.2)$ is a special case of the maximin formula (1.1).

If $T$ is an order preserving and positively sub-homogeneous self-map of the interior of a (closed, convex and pointed) cone $C$ in a finite dimensional vector space, we define the radial extension $\hat{T}$ of $T$ to the closed cone $C$ by

$$
\hat{T}(x):=\lim _{\epsilon \rightarrow 0^{+}} T(x+\epsilon z), \quad \forall x \in C
$$

where $z$ is any point in the interior of $C$. One verifies that this definition is independent of the choice of $z$; equivalently, we may define $\hat{T}(x)$ as $\inf \{T(y) \mid y-x \in \operatorname{int} C\}$. The order preserving and positively (sub-)homogeneous character of $T$ carries over to $\hat{T}$. It is shown in BNS03] that $T$ does not necessarily have a continuous extension to $C$, but that it always does if $C$ is polyhedral. 
Lemma 36. Let $T$ be an order-preserving and positively sub-homogeneous self-map of the interior of a (convex closed and pointed) cone $C$ in a finite dimensional vector space, and consider the Martin function $h$ in (4.3) associated to a vector $u \in C \backslash\{0\}$. Then, for all $\lambda \in \mathbb{R}$,

$$
(\forall x \in \operatorname{int} C, h(T(x))-h(x) \geqslant \lambda) \Longleftrightarrow(\forall \gamma>0, \hat{T}(\gamma u) \geqslant \gamma \exp (\lambda) u)
$$

Proof. Since, $h(T(x))-h(x)=-\operatorname{RFunk}(T(x), u)+\operatorname{RFunk}(x, u)$, the left-hand side condition in 4.7 holds if and only if

$$
u \leqslant \exp (-\lambda+\operatorname{RFunk}(x, u)) T(x), \quad \forall x \in \operatorname{int} C .
$$

For $\epsilon>0$ and $\gamma>0$, define $x_{\epsilon}:=\gamma u+\epsilon z$, where $z$ is a given element in $\operatorname{int} C$. Then, $\operatorname{RFunk}\left(x_{\epsilon}, u\right) \leqslant \operatorname{RFunk}(\gamma u, u)=-\log \gamma$. Taking $x=x_{\epsilon}$ in (4.8), and letting $\epsilon$ tend to zero, we get

$$
u \leqslant \exp (-\lambda) \gamma^{-1} \hat{T}(\gamma u)
$$

and so, the condition at the right-hand side of (4.7) holds. Conversely, fix $x \in \operatorname{int} C$, and choose $\gamma$ such that $\gamma \operatorname{RFunk}(x, u) \geqslant 1$. We have

$$
\begin{array}{rlr}
\gamma u & \leqslant \exp (-\lambda) \hat{T}(\gamma u) \quad \text { (using the right-hand side of }(4.7)) \\
& \leqslant \exp (-\lambda) \hat{T}(\gamma \exp (\operatorname{RFunk}(x, u)) x) \quad \text { (since } \hat{T} \text { is order preserving) } \\
& \leqslant \exp (-\lambda) \gamma \exp (\operatorname{RFunk}(x, u)) \hat{T}(x) \quad \text { (since } \hat{T} \text { is sub-homogeneous) } \\
& =\gamma \exp (-\lambda+\operatorname{RFunk}(x, u)) T(x) .
\end{array}
$$

Cancelling $\gamma$, we arrive at 4.8).

Observe that when $T$ is positively sub-homogeneous, the following recession map

$$
\hat{T}_{r}: C \rightarrow C, \quad \hat{T}_{r}(x)=\lim _{\gamma \rightarrow \infty} \gamma^{-1} \hat{T}(\gamma x)=\inf _{\gamma>0} \gamma^{-1} \hat{T}(\gamma x)
$$

is well defined. Of course, $\hat{T}=\hat{T}_{r}$ if $T$ is positively homogeneous. We now arrive at the following corollary of Theorem 1, which extends the Collatz-Wielandt formula (1.2).

Corollary 37. Let $T$ be an order-preserving and positively sub-homogeneous self-map of the interior of a symmetric cone in a finite dimensional vector space, and assume that

$$
\rho(T):=\lim _{k \rightarrow \infty} \frac{\log \operatorname{RFunk}\left(x, T^{k}(x)\right)}{k}>0 .
$$

Then,

$$
\inf _{y \in \operatorname{int} C} \max _{w \in \operatorname{Extr} C} \log \frac{\langle w, T(y)\rangle}{\langle w, y\rangle}=\rho(T)=\max _{u \in C \backslash\{0\}} \min _{\substack{w \in \operatorname{Extr} C \\\langle w, u\rangle \neq 0}} \log \frac{\left\langle w, \hat{T}_{r}(u)\right\rangle}{\langle w, u\rangle} .
$$

Moreover, when $T$ is positively homogeneous, the same conclusion remains valid even when the condition $\rho(T)>0$ does not hold. 
Proof. We apply Theorem 16 to the space $X:=\operatorname{int} C$ equipped with the hemi-metric $\delta:=$ RFunk $^{+}$. Let us denote by $\rho_{+}(T)$ the escape rate of $T$ in this hemi-metric, so that

$$
\rho_{+}(T)=\inf _{y \in \operatorname{int} C} \operatorname{RFunk}^{+}(y, T(y))
$$

Since $\mathrm{RFunk}^{+}=\max (\mathrm{RFunk}, 0)$, the hemi-metrics RFunk and RFunk ${ }^{+}$coincide as soon as RFunk ${ }^{+}$or RFunk is positive. Hence, $\rho(T)>0$ implies that $\rho_{+}(T)=\rho(T)$ and that the term $\operatorname{RFunk}^{+}(y, T(y))$ in 4.12 can be replaced by $\operatorname{RFunk}(y, T(y))$. Together with (4.1), this gives the first equality in (4.11).

We observed in the proof of Corollary 33 that when the escape rate in the RFunk ${ }^{+}$ hemi-metric is positive, the corresponding horofunction $h$ in Theorem 16 is necessarily of the form (4.3). Since the limit coincides with the infimum in the definition of the recession function (see (4.9)), the right-hand side condition in (4.7) is equivalent to

$$
\hat{T}_{r}(u) \geqslant \exp (\lambda) u
$$

Then, using Lemma 36, we rewrite the maximin characterisation in Theorem 16 as

$$
\begin{aligned}
\rho(T) & =\max _{h} \inf _{x \in \operatorname{int} C} h(T(x))-h(x) \\
& =\max \left\{\lambda \mid \exists u \in C \backslash\{0\}, \hat{T}_{r}(u) \geqslant \exp (\lambda) u\right\} \\
& \left.=\max _{u \in C \backslash\{0\}} \log \inf _{w \in \operatorname{Extr} C\langle w, u\rangle \neq 0} \frac{\left\langle w, \hat{T}_{r}(u)\right\rangle}{\langle w, u\rangle} \quad \text { (by } 4.2\right) .
\end{aligned}
$$

Moreover, it follows from Remark 30 that the latter infimum is attained.

Finally, when $T$ is positively homogeneous, we equip the space int $C$ with the hemimetric RFunk instead of RFunk ${ }^{+}$. Then, the first equality in 4.11 follows readily from Theorem 16 and (4.1) (the assumption that $\rho(T)>0$ is not needed any more), and the proof of the second inequality is unchanged.

Remark 38. The characterisation 4.11 can be rewritten equivalently as

$$
\begin{aligned}
\rho(T) & =\log \inf \{\mu>0 \mid \exists y \in \operatorname{int} C, T(y) \leqslant \mu y\} \\
& =\log \max \left\{\mu \geqslant 0 \mid \exists u \in C \backslash\{0\}, \hat{T}_{r}(u) \geqslant \mu u\right\} .
\end{aligned}
$$

Remark 39. When $C$ is the standard positive cone, and $T$ is a continuous order preserving and positively homogeneous self-map of the closed cone $C$, Nussbaum [Nus86] showed that

$$
\rho(T)=\log \max \{\mu \geqslant 0 \mid \exists u \in C \backslash\{0\}, T(u)=\mu u\},
$$

which is more accurate than (4.14). Moreover, some of the results of [Nus86 have been extended in [MPN02 to more general (normal, possibly infinite dimensional) cones. However, Corollary 37 is applicable when $T$ is only defined on the interior of the cone and does not extend continuously to its boundary (this situation does occur in practice, see the discussion in BNS03 of the matrix harmonic mean). 
Example 40. We finally illustrate the previous Denjoy-Wolff type results with an example originating from quadratic optimal control. Let $A, B$ be $n \times n$ positive semidefinite matrices, and let $M$ be a $n \times n$ invertible matrix. Consider the following discrete time Riccati operator, which is a self-map of the interior of the cone $S_{n}^{+}$of $n \times n$ positive semidefinite symmetric matrices,

$$
T(X)=A+M\left(B+X^{-1}\right)^{-1} M^{*},
$$

where $M^{*}$ denotes the transpose of $M$. We refer the reader to [Bou95, LW94, LL08] for more background on these maps. In particular, $T$ is order-preserving, and, as shown by Liverani and Wojtkowski |LW94|, $T$ is non-expansive in the Thompson metric. We noted above that the latter property, together with the preservation of order, implies that $T$ is positively sub-homogeneous, and so, Corollaries 33 and 37 apply to the map $T$.

If the matrices $A$ and $B$ are positive definite, then $T$ is known to be a strict contraction in Thompson metric [LW94]. It follows that $T$ has a unique fixed point in int $S_{n}^{+}$to which every orbit converges. In particular, the escape rate $\rho(T)$ is zero.

We next examine a more interesting case in which $B$ is of rank one, leading to a positive escape rate. Assume that $n=2$, write $B=v v^{*}$ for some (column) vector $v$, and assume that $M$ is of the form $M=\alpha I$, with $\alpha>1$, where $I$ is the identity matrix.

We claim that $\rho(T)=2 \log \alpha$. To see this, let $u$ denote a non-zero vector orthogonal to $v$, and consider $U=u u^{*}$. Then, it is easily checked that the radial extension of $T$ satisfies $\hat{T}(\gamma U)=A+\alpha^{2} \gamma U$ for all $\gamma>0$, and so, $\hat{T}_{r}(U)=\alpha^{2} U$. Using (4.14), we deduce that $\rho(T) \geqslant 2 \log \alpha$. To show that the equality holds, we observe that $T(X) \leqslant A+M X M^{*}$. Hence, for all $s>0, T(s I) \leqslant A+\alpha^{2} s I \leqslant\left(\lambda_{1}(A) s^{-1}+\alpha^{2}\right) s I$, where $\lambda_{1}(A)$ denotes the maximal eigenvalue of $A$, which by (4.13), implies that $\rho(T) \leqslant \log \left(\lambda_{1}(A) s^{-1}+\alpha^{2}\right)$. Letting $s$ tend to infinity, we arrive at $\rho(T) \leqslant 2 \log \alpha$, and so $\rho(T)=2 \log \alpha$.

A possible choice of the horofunction $h$ appearing in Theorem 16 is

$$
h(X)=-\operatorname{RFunk}(X, U)+\operatorname{RFunk}(I, U),
$$

where the basepoint is the identity matrix $I$. Then, the linear form $x \mapsto\langle w, x\rangle$ constructed in the proof of Corollary 33 can be checked to be $X \mapsto\langle U, X\rangle$ (or any scalar multiple of this form).

For $n=2$, the cone of positive semidefinite matrices $S_{n}^{+}$has the shape of a Lorentz (ice-cream) cone. Then, the superlevel set $h(X) \geqslant \lambda$ is nothing but the interior of the translated Lorentz cone $\exp (\lambda-\operatorname{RFunk}(I, U)) U+S_{2}^{+}$. This is illustrated in Figure 1.

Acknowledgements. This work originates from discussions of Brian Lins and the first author at the AIM workshop on non-negative matrix theory in Palo Alto in 2008, where they jointly obtained an early version of Theorem 16 concerning the special Banach space case. The first author thanks Brian Lins for these discussions, and also for more recent observations.

\section{References}

[AB06] C.D. Aliprantis and K.C. Border. Infinite dimensional analysis. Springer, 2006. 


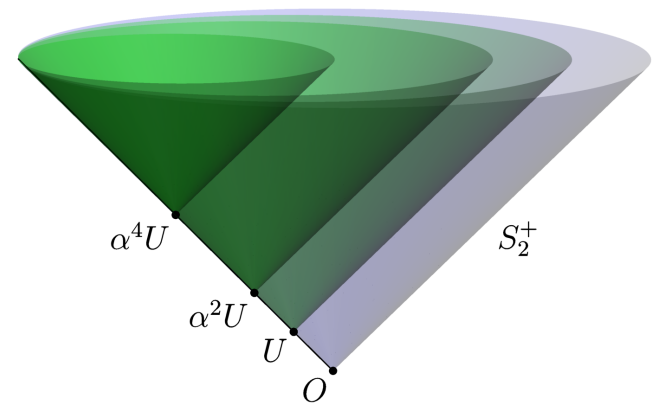

Figure 1: Theorem 16 applied to a discrete Riccati operator on the cone of positive definite matrices int $S_{2}^{+}$(Example 40). The horoballs (superlevel sets) of the horofunction $h$ are nested cones (in green) intersecting the cone $S_{2}^{+}$(in blue) along the ray generated by the matrix $U$. The map $T$ sends every horoball $h \geqslant \lambda$ to the horoball $h \geqslant \lambda+\rho(T)$. Three such horoballs are represented, with apices $U, \alpha^{2} U, \alpha^{4} U$, corresponding to $\lambda=0, \rho(T), 2 \rho(T)$, respectively.

[ACS00] E. Andruchow, G. Corach, and D. Stojanoff. Geometrical significance of Löwner-Heinz inequality. Proc. Amer. Math. Soc., 128(4):1031-1037, 2000.

[AGLN06] M. Akian, S. Gaubert, B. Lemmens, and R. Nussbaum. Iteration of order preserving subhomogeneous maps on a cone. Math. Proc. Cambridge Philos. Soc., 140(01):157-176, 2006.

[AGW09] M. Akian, S. Gaubert, and C. Walsh. The max-plus Martin boundary. Doc. Math., 14:195-240, 2009.

[Bal95] W. Ballmann. Lecture on spaces of nonpositive curvature. Birkhäuser, 1995.

[Bea97] A. F. Beardon. The dynamics of contractions. Ergodic Theory Dynam. Systems, 17(6):1257-1266, 1997.

[BGS85] W. Ballmann, M. Gromov, and V. Schroeder. Manifolds of nonpositive curvature, volume 61 of Progress in Mathematics. Birkhäuser Boston Inc., Boston, MA, 1985.

[Bha03] R. Bhatia. On the exponential metric increasing property. Linear Algebra Appl., 375:211-220, 2003.

[BNS03] A. D. Burbanks, R. D. Nussbaum, and C. T. Sparrow. Extension of orderpreserving maps on a cone. Proc. Roy. Soc. Edinburgh Sect. A, 133(1):35-59, 2003.

[Bou95] Ph. Bougerol. Almost sure stabilizability and Riccati's equation of linear systems with random parameters. SIAM J. Control Optim., 33(3):702-717, 1995. 
[Bow08] R. Bowen. Equilibrium states and the ergodic theory of Anosov diffeomorphisms, volume 470 of Lecture Notes in Mathematics. Springer-Verlag, Berlin, revised edition, 2008.

[Den26] A. Denjoy. Sur l'itération des fonctions analytiques. C.R. Acad. Sci. Paris, 182:255-257, 1926.

[FF04] S. Friedland and P. J. Freitas. $p$-metrics on $\mathbf{G L}(m, \mathbb{C}) / \mathbf{U}_{n}$ and their busemann compactifications. Linear Algebra Appl., 376:1-18, 2004.

[FK94] J. Faraut and A. Korányi. Analysis on symmetric cones. Oxford Mathematical Monographs. The Clarendon Press Oxford University Press, New York, 1994.

[Fun29] P. Funk. Über Geometrien, bei denen die Geraden die Kürzesten sind. Math. Ann., 101(1):226-237, 1929.

[GG04] S. Gaubert and J. Gunawardena. The Perron-Frobenius theorem for homogeneous, monotone functions. Trans. Amer. Math. Soc., 356(12):4931-4950, 2004.

[GK95] J. Gunawardena and M. Keane. On the existence of cycle times for some nonexpansive maps. Technical report, Citeseer, 1995.

[Gro81] M. Gromov. Hyperbolic manifolds, groups and actions. In Riemann surfaces and related topics: Proceedings of the 1978 Stony Brook Conference (State Univ. New York, Stony Brook, N.Y., 1978), volume 97 of Ann. of Math. Stud., pages 183-213, Princeton, N.J., 1981. Princeton Univ. Press.

[Gun03] J. Gunawardena. From max-plus algebra to nonexpansive maps: a nonlinear theory for discrete event systems. Theoret. Comput. Sci., 293(1):141-167, 2003.

[GW03] J. Gunawardena and C. Walsh. Iterates of maps which are non-expansive in Hilbert's projective metric. Kybernetika (Prague), 39(2):193-204, 2003. Special issue on max-plus algebras (Prague, 2001).

[IM07] H. Ishii and H. Mitake. Representation formulas for solutions of HamiltonJacobi equations with convex Hamiltonians. Indiana Univ. Math. J., 56(5):2159-2183, 2007.

[Kar01] A. Karlsson. Non-expanding maps and Busemann functions. Ergodic Theory Dynam. Systems, 21(5):1447-1457, 2001.

[KMN06] A. Karlsson, V. Metz, and G. A. Noskov. Horoballs in simplices and Minkowski spaces. Int. J. Math. Math. Sci., pages Art. ID 23656, 20, 2006.

[KN81] E. Kohlberg and A. Neyman. Asymptotic behavior of nonexpansive mappings in normed linear spaces. Israel J. Math., 38(4):269-275, 1981. 
[Lin07] B. Lins. A Denjoy-Wolff theorem for Hilbert metric nonexpansive maps on polyhedral domains. Math. Proc. Cambridge Philos. Soc., 143(01):157-164, 2007.

[Lin09] B. Lins. Asymptotic behavior of nonexpansive mappings in finite dimensional normed spaces. Proc. Amer. Math. Soc., 137(7):2387-2392, 2009.

[LL07a] J. Lawson and Yongdo Lim. A Birkhoff contraction formula with applications to Riccati equations. SIAM J. Control Optim., 46(3):930-951, 2007.

[LL07b] J. Lawson and Yongdo Lim. Metric convexity of symmetric cones. Osaka J. Math., 44(4):795-816, 2007.

[LL08] Hosoo Lee and Yongdo Lim. Invariant metrics, contractions and nonlinear matrix equations. Nonlinearity, 28:857-878, 2008.

[LS05] B. Lemmens and M. Scheutzow. On the dynamics of sup-norm nonexpansive maps. Ergodic Theory Dynam. Systems, 25(3):861-871, 2005.

[LW94] C. Liverani and M. P. Wojtkowski. Generalization of the Hilbert metric to the space of positive definite matrices. Pacific J. Math., 166(2):339-355, 1994.

[Mos55] G.D. Mostow. Some new decomposition theorems for semisimple groups. Memoirs Amer. Math. Soc., 14:31-54, 1955.

[MPN02] J. Mallet-Paret and Roger Nussbaum. Eigenvalues for a class of homogeneous cone maps arising from max-plus operators. Discrete Contin. Dyn. Syst., 8(3):519-562, 2002.

[MSZ94] J.-F. Mertens, S. Sorin, and S. Zamir. Repeated games. core reprint dps 9420, 9421 and 9422. Center for Operation Research and Econometrics, Universite Catholique De Louvain, Belgium, 1994.

[Ney03] A. Neyman. Stochastic games and nonexpansive maps. In Stochastic games and applications (Stony Brook, NY, 1999), volume 570 of NATO Sci. Ser. C Math. Phys. Sci., pages 397-415. Kluwer Acad. Publ., Dordrecht, 2003.

[Now03] A.S. Nowak. Zero-sum stochastic games with Borel state spaces. Stochastic games and applications, page 77, 2003.

[NS04] A. Neyman and S. Sorin. Stochastic games and applications. Kluwer Academic Publishers, 2004.

[Nus86] R. D. Nussbaum. Convexity and log convexity for the spectral radius. Linear Algebra Appl., 73:59-122, 1986.

[Nus88] R. D. Nussbaum. Hilbert's projective metric and iterated nonlinear maps. Mem. Amer. Math. Soc., 75(391):iv+137, 1988. 
[Nus94] R. D. Nussbaum. Finsler structures for the part metric and hilbert's projective metric and applications to ordinary differential equations. Differential and Integral Equations, 7(6):1649-1707, 1994.

[Nus07] R. D. Nussbaum. Fixed point theorems and Denjoy-Wolff theorems for Hilbert's projective metric in infinite dimensions. Topol. Methods Nonlinear Anal., 29(2):199-249, 2007.

[NW04] R. D. Nussbaum and C. Walsh. A metric inequality for the Thompson and Hilbert geometries. J. Inequalities Pure Appl. Math., 5(3), 2004.

[Pap05] A. Papadopoulos. Metric spaces, convexity and nonpositive curvature. European Mathematical Society, 2005.

[Paz71] A. Pazy. Asymptotic behavior of contractions in Hilbert space. Israel J. Math., 9(2):235-240, 1971.

[PT09] A. Papadopoulos and M. Troyanov. Weak Finsler structures and the Funk weak metric. Math. Proc. Cambridge Philos. Soc., 147(2):419-437, 2009.

[Rei73] S. Reich. Asymptotic behavior of contractions in Banach spaces. J. Math. Anal. Appl., 44:57-70, 1973.

[Rie02] M. A. Rieffel. Group $C^{*}$-algebras as compact quantum metric spaces. Doc. Math., 7:605-651, 2002.

[RS01] D. Rosenberg and S. Sorin. An operator approach to zero-sum repeated games. Israel J. Math., 121(1):221-246, 2001.

[RS05] S. Reich and D. Shoikhet. Nonlinear semigroups, fixed points, and geometry of domains in Banach spaces. Imperial College Press, London, 2005.

[Sha53] L.S. Shapley. Stochastic games. Proceedings of the National Academy of Sciences of the United States of America, 39(10):1095, 1953.

[Wal07] C. Walsh. The horofunction boundary of finite-dimensional normed spaces. Math. Proc. Cambridge Philos. Soc., 142(3):497-507, 2007.

[Wal08] C. Walsh. The horofunction boundary of the Hilbert geometry. Adv. Geom., 8(4):503-529, 2008.

[Wol26a] J. Wolff. Sur l'itération des fonctions bornées. C.R. Acad. Sci. Paris, 182:200201, 1926.

[Wol26b] J. Wolff. Sur une généralisation d'un théorème de Schwarz. C.R. Acad. Sci. Paris, 182-183:918-920, 500-502, 1926. 